\title{
Video Watermarking using DWT and Alpha Blending Technique
}

\author{
Asha $\mathbf{N}^{1}$, Bhagya $\mathbf{P}^{2}$ \\ Department of Electronics and Communication, DBIT, VTU, Belagauvi, Karnataka, India ${ }^{1}$ \\ Associate Professor, Department of Electronics and Communication, DBIT, VTU, Belagauvi, Karnataka, India ${ }^{2}$
}

\begin{abstract}
In cyber world, protection of copyrighted information is catching its attention due to the accessibility of immense amount of data and its copies in digital form. An efficient method of protecting this copyrighted information is by Digital Watermarking. In this paper, Discrete Wavelet Transform (DWT) and Alpha Blending Techniques are used. The video is converted into frames and DWT is applied on the frames and the watermark, alpha blending technique is applied to embed the watermark in the video frames. Finally Alpha blending extracting technique and inverse discrete wavelet transform is used to extract the watermark from the watermarked video. The robustness of the proposed method is analyzed by PSNR and MSE values.
\end{abstract}

Keywords: Digital Video Watermarking, Discrete Wavelet Transform (DWT), Alpha Blending Technique, PSNR, MSE.

\section{INTRODUCTION}

In today's world there is a rapid growth of internet technology and multimedia products such as image, audio, videos etc. the security and authenticity issues are becoming popular. Watermarking is a concept of embedding special symbol or $\log$ o or pattern watermark, in to video so that some copyright information is fed into it. This information can inform users about rights holder or authority to permit the use of data, it can later prove for ownership, identify misappropriate persons, tracking of the video [1]. Many researchers and technologies were proposed to provide methods to solve the problem of illegal copies and manipulation of digital video. An attractive method that has been proposed to implement copyright information and authenticating in digital video is digital watermarking $[1,2]$. Watermarking can be classified into two main categories based on the method of hiding watermark information bits in the host video. The two categories are: Spatial domain watermarking and transform-domain watermarking [8, 9]. In spatial-domain watermarking techniques, embedding and detection are performed on spatial pixels values (luminance, chrominance, and color space) or on the overall video frame. Spatial-domain techniques are easy to implement, however they are not robust against common digital signal processing operations such as video compression. Transform-domain techniques, on the other hand, alter spatial pixel values of the host video according to a pre-determined transform. Commonly used transforms are the Discrete Cosine Transform (DCT), the Fast Fourier transform (FFT), the Discrete Wavelet Transform (DWT), and the Singular Value Decomposition (SVD). Transform-domain watermarking techniques proved to be more robust and imperceptible compared to spatial domain techniques since disperse the watermark in the special domain of video frame, making it very difficult to remove the embedded watermark. The watermarking may be visible or invisible. In this paper we proposed an visible watermarking technique based on 3 level DWT. DWT is more computationally efficient than other transform methods like DFT and DCT. Due to its excellent spatial-frequency localization properties, the DWT is very suitable to identify areas in the host video frame where a watermark can be embedded imperceptibly. It is known that even after the decomposition of the video frame using the wavelet transformation there exist some amount of correlation between the wavelet coefficients $[4,5,6]$. Watermarking scheme quality is determined using robustness, transparency and capacity. Transparency means after insertion of watermark the original image should not be distorted Robustness is related to attacks. If watermark removal is difficult to various attacks like rotation, scaling, compression, noise then watermarking scheme is robust. Capacity means amount of information inserted to image. More capacity means one can hide large amount of information [6, 7].

\section{EXISTING WORK}

Vivek Kumar Agrawal [10] has proposed a static 3D-DCT scheme which is implemented on correlated video. It is observed that this scheme gives favorable results for low motion activity videos but produces visual distortion in high motion activities.

G. Langelaar [11] has proposed a watermarking scheme in w3hich a watermark is embedded by adding pseudorandom noise to image components and detected by correlating the pseudorandom noise with the image components. 
Vivek Agarwal and Sumana Gupta [12], has proposed a watermarking scheme in which a variable-length 3-D DCT is chosen to exploit the redundancy of the video sequences in temporal domain. The method adopted embeds the watermark (binary logo image) in an uncompressed video sequence by modifying the values of the mid-range coefficients of 3-D DCT block, chosen in accordance with the 3-D human visual system model which preserves the perceptual quality of video sequences. The experimental results demonstrate the robustness of the proposed watermarking procedure to several video degradations and watermarking attacks.

M. Carli1, C. Adsumilli, V. Razzauti, and A. Neri [13], authors have implemented three different methods for watermarking a video in 3D-DCT domain. These spatiotemporal volume embedding techniques have been proved to be very effective in embedding a huge amount of data with little or no visible distortion to the host video.

Gu Tiaming and Wang Yangie [14] proposed the robust image watermarking algorithm. This algorithm is based on DWT coefficient. The level-3 DWT is done to obtain the low frequency part and the information in this part is used along with watermark to obtain the key. The key generated is used to extract the watermark. This algorithm does not change the content of original image and thus robust to various attacks.

Hong, Kim and Han [15] proposed a watermarking scheme in which watermark is embedded in middle frequency band of the two level DWT. They have generated the pseudo random code which is used for watermark positioning in the LH2 (level-2 LH component) band. The mean value of neighboring pixel is found out and this mean value is then compared with the selected pixel. Replacement of selected pixel is done based on the comparison and new flags are generated consisting of values 0 and 1 based on the conditions suggested by the authors. This generated flag is stored and used for watermark extraction.

Daren, Jiufen, Jiwu and Hongmie [16] embed watermark in low frequency sub-band first and remaining in higher frequency sub bands depending on the significance of sub band. Watermark embedding is done with different embedding formulae. This algorithm incorporates the features of visual masking of human vision system in watermarking.

\section{DISCRETE WAVELET TRANSFORM}

The use of Wavelet transform will mainly address the capacity and robustness of the Information- Hiding system features. The Discrete Wavelet Transform is the simplest of all wavelet transform. In this the low frequency wavelet coefficients are generated by averaging the two pixel values and high frequency coefficients that are generated by taking half of the difference of the same two pixels. The four bands obtained are LL, LH, HL, and HH which is shown in Fig 1. The LL band is called as approximation band, which consists of low frequency wavelet coefficients, and contains significant part of the spatial domain image. The other bands are called as detail bands which consist of high frequency coefficients and contain the edge details of the spatial domain image.

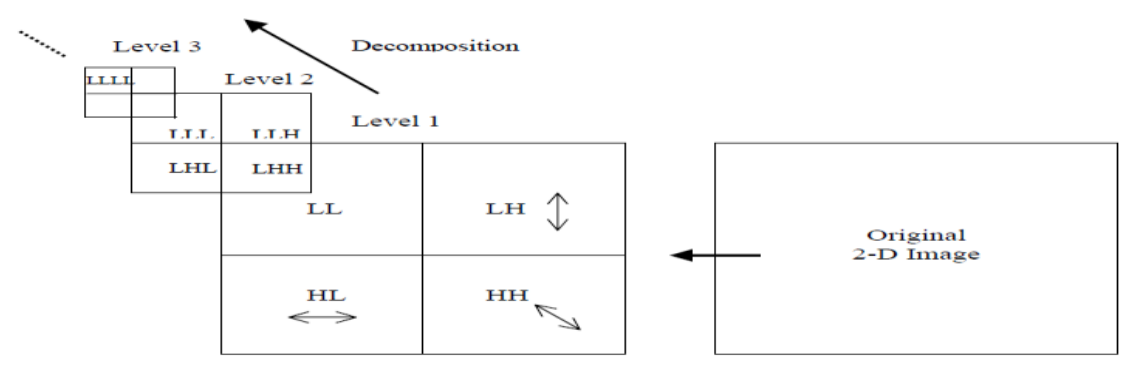

Fig.1 3-level DWT

\section{ALPHA BLENDING TECHNIQUE}

Alpha Blending can be accomplished by blending every pixel from the primary source image (cover image) with the relating pixel in the second source image (watermark).

According to the formula of the alpha blending the watermarked image is given by

$$
\mathbf{W M I}=\mathbf{k} * \mathbf{L} \mathbf{L}+\mathbf{q} * \mathbf{W M}
$$

Where,

WMI = Low frequency (recurrence) segment of the watermarked image

$\mathbf{L L}=$ Low frequency (recurrence) segment of the cover image,

$\mathbf{W M}=$ Low frequency (recurrence) component of watermark,

$\mathbf{k}$ and $\mathbf{q}=$ scaling factors for cover image and watermark image respectively. 
According to formula of alpha blending the recovered image is given by

$$
\mathbf{R W}=(\mathbf{W M I}-\mathbf{k} * \mathbf{L L})
$$

Where,

$\mathbf{R W}=$ Low frequency segment of recovered watermark,

WMI = Low frequency segment of watermarked image, $\mathbf{k}=$ scaling factor for cover image.

\section{PROPOSED METHOD}

In this paper we have proposed 3-level DWT based video Watermarking. Fig. 2 shows the watermark embedding scheme within the video frame. The input video is converted into frames and DWT is applied at 3 levels as shown in Fig 1 both on watermark and the video frames. Alpha Blending Technique is applied to embed the watermark within the input frames and finally IDWT is applied to get back the frames. The frames are then converted back to get back the watermarked video.
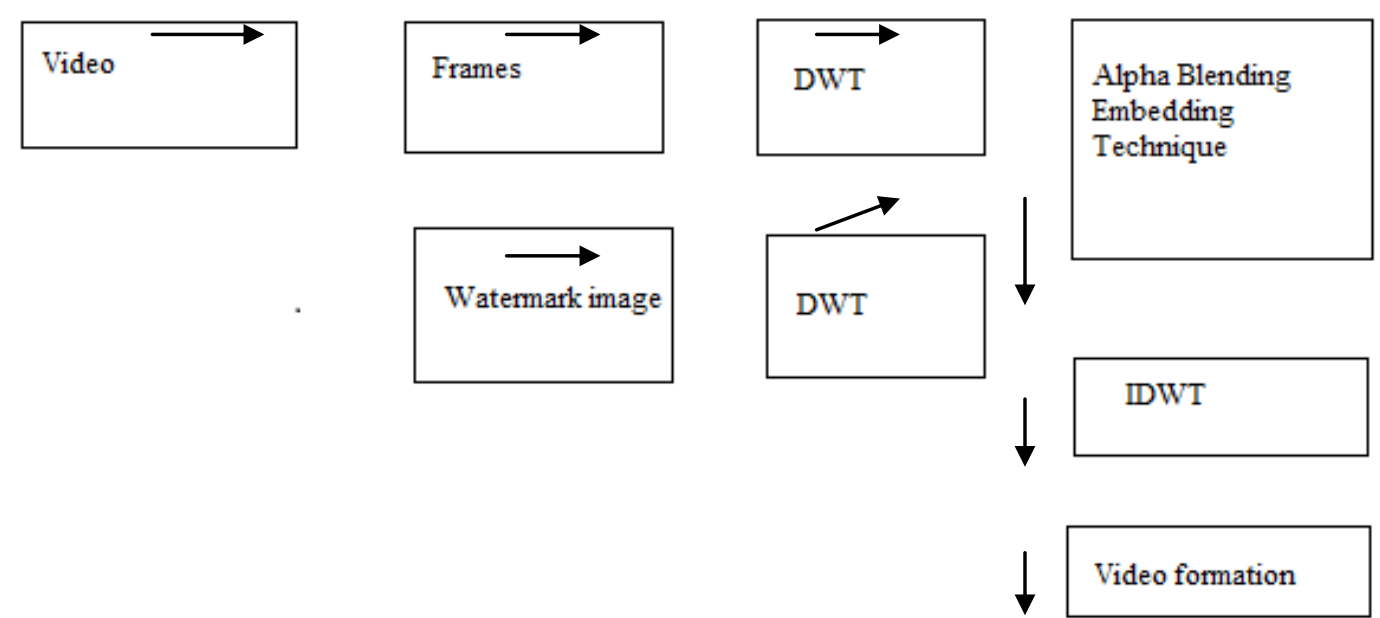

\section{Video formation}

Watermarked video

Fig. 2 Watermark Embedding Scheme

Fig. 3 shows the Watermark Extracting scheme in which the watermarked video is converted into frames and DWT is applied on the frames. Alpha Blending extraction technique is applied on cover video and LL sub band of Watermarked frame to extract the watermark.
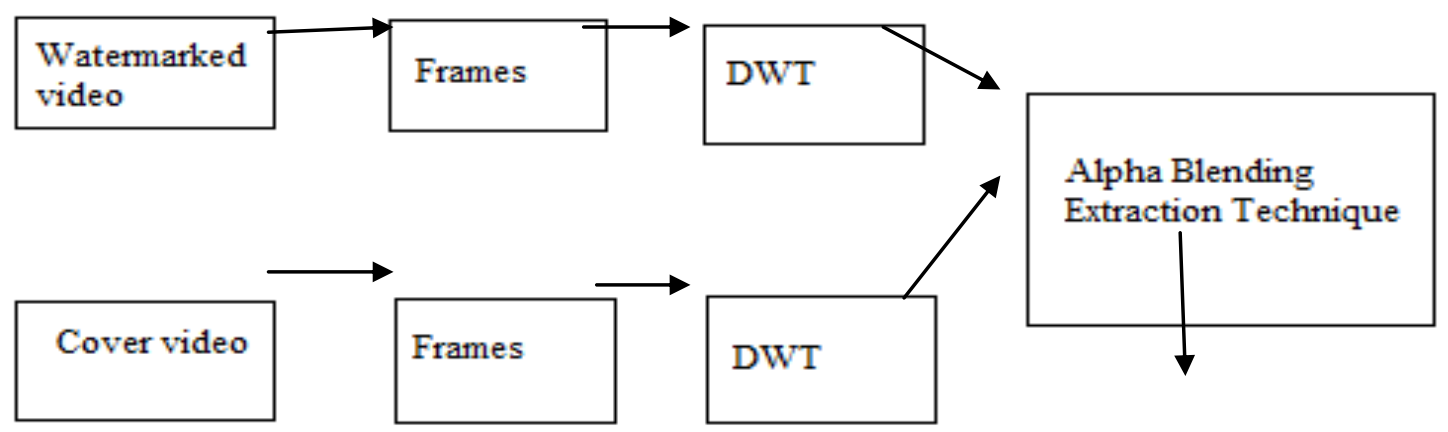

Extracted Watermark

Fig. 3 Watermark Extracting Scheme 


\section{EXPERIMENTAL RESULTS}

The proposed watermarking algorithm is simulated using MATLAB R2014a. The proposed watermarking algorithm is tested for input video consisting of 200 frames and watermark image (fingerprint) of size 512*512. The performance of the proposed method is evaluated by PSNR (Peak Signal to Noise Ratio) and MSE (Mean Square Error). PSNR is used to measure the imperceptibility between the original image and watermarked image. PSNR is defined by the eqn. (3). The similarity between the original watermark and the extracted watermark from the attacked image is evaluated by using MSE given by eqn. (4).

$$
\begin{aligned}
& \operatorname{PSNR}(\mathbf{d b})=10 * \log \left(\frac{255^{2}}{\operatorname{MSE}}\right) \\
& \operatorname{MSE}=\sum_{\mathrm{i}=1}^{\mathrm{x}} \sum_{\mathrm{j}=1}^{\mathrm{y}} \frac{\left(\left|\mathrm{A}_{\mathrm{ij}}-\mathbf{B}_{\mathrm{ij}}\right|\right)^{2}}{\mathrm{x} * \mathrm{y}}
\end{aligned}
$$

Where, $\mathbf{x}, \mathbf{y}=$ size of the watermark image.

A = original watermark image.

B = extracted watermark image.

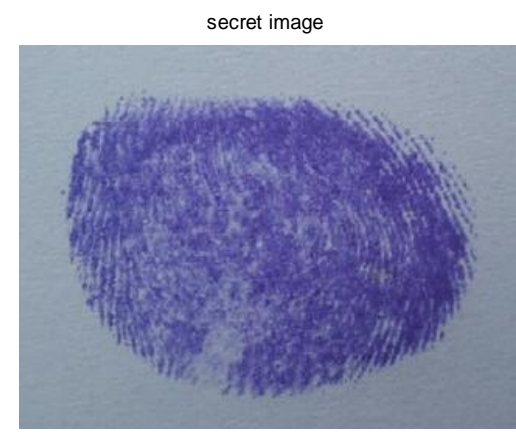

(a)

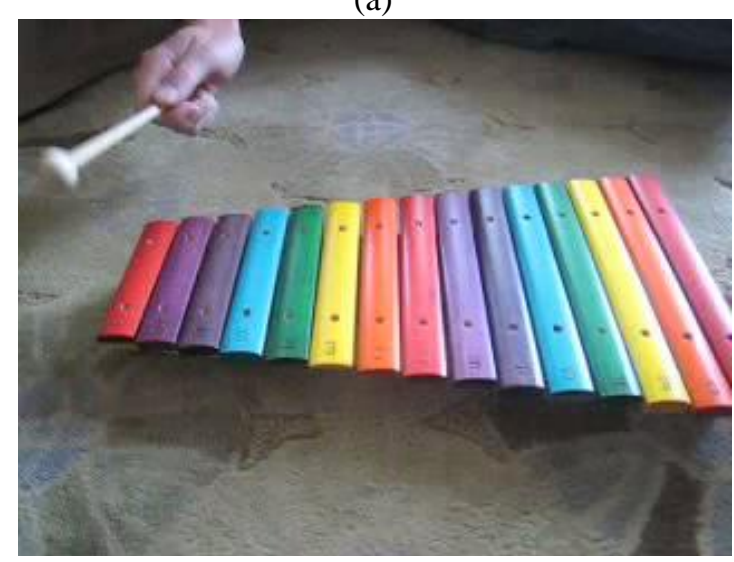

(b)

watermarked image

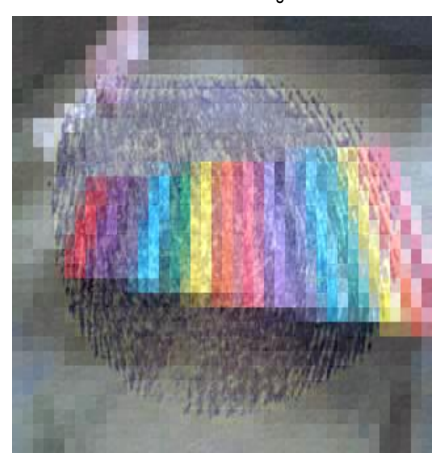

(C) 


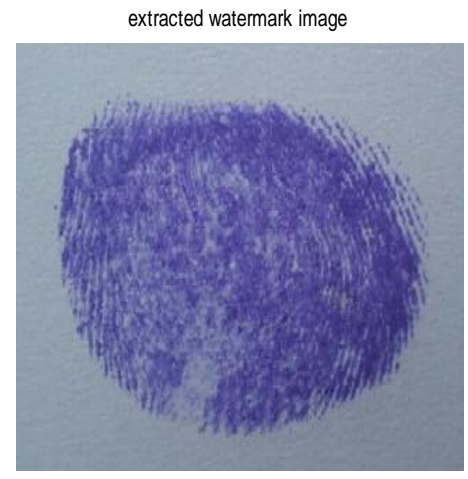

(d)

Fig.4 (a) Watermark (b) Input video (c) watermarked video (d) Extracted watermark

Table.1 PSNR and MSE values for different scaling factors

\begin{tabular}{|l|l|l|l|l|}
\hline Number of frames & P value & Q value & PSNR & MSE \\
\hline 5 & 0.9 & 0.2 & 91.114 & 2.0280 \\
\hline 20 & 0.88 & 0.3 & 91.150 & 2.0113 \\
\hline
\end{tabular}

\section{CONCLUSION}

A DWT based video watermarking technique has been implemented and the watermark is embedded and extracted from the cover image by alpha blending technique. The quality of the watermark depends on the scaling factor. The quality of original and the recovered watermark are compared by calculating the PSNR and MSE values. The simulation results show that the PSNR values are comparatively high compared to the other approach. This method is more robust to attacks.

\section{REFERENCES}

[1] Snehal V. Patel and Prof Arvind R. watermarking using 4-level DWT", Natioanl conference on resent trends in engineering and technology 2011.

[2] Anitha \& R. Leela Velusamy, Authenticating of digital document using secret key biometric watermarking" International journal of communication network security 2012.

[3] Hae Yong Kim and Ricardo L.de Queiroz,"A public key authentication watermarking for binary images" University of Brazil.

[4] Tamanna Tabassum and S.M.Mohidul Islam, "A Digital Video Watermarking Technique Based on Identical Frame Extraction in 3-Level DWT" IEEE, 2012.

[5] Thanikaiselvan.V, Arulmozhivarman.P and Rengarajan Amirtharajan, (e) John Bosco Balaguru Rayappan, Wave(Let) Decide Choosy Pixel Embedding for Stego", International Conference on computer communication and electrical technology, ICCCET 2011.

[6] Nidhi Divecha and Dr. N. N. Jani,'Implementation and performance analysis of DCT-DWT-SVD based watermarking algorithms for color images", 2013 International Conference on Intelligent Systems and Signal Processing (ISSP).

[7] Pik Wah Chan, Student Member, IEEE, Michael R. Lyu, Fellow, IEEE, and Roland T. Chin, "A Novel Scheme for Hybrid Digital Video Watermarking: Approach, Evaluation and Experimentation”, IEEE Transactions on circuits and systems for video technology, vol. 15, NO. 12, December 2005.

[8] G. Doerr and J. Dugelay, “A guide tour of video watermarking,” Signal processing: Image communication, vol. 18, no. 4, pp. $263-282$, 2003.

[9] You-Ru Lin, Hui-Yu Huang, and Wen-Hsing Hsu, “An embedded watermark technique in copyright proctection", International conference on Pattern Recognition (ICPR 06).

[10] Vivek Kumar Agrawal on "Perceptual Watermarking Of Digital Video Using Variable Temporal Length 3D- DCT “, Thesis, Department of electrical engg, IIT Kanpur,2007.

[11] G. Langelaar, I. Setyawan, R. Lagendijk, "Watermarking Digital Image and Video Data”, in IEEE Signal Processing Magazine, Vol. 17, Sept. 2000, pp. 20-43.

[12] Vivek Agarwal and Sumana Gupta," Variable temporal length 3D-DCT based video watermarking", 20th Annual IS\&T/SPIE Symposium on Electronic Imaging, 27-31 January 2008, San Jose, California USA .

[13] M. Carli1, C. Adsumilli, V. Razzauti, and A. Neri, "Video watermarking In 3D-DCT Domain", 14th European Signal Processing Conference (EUSIPCO 2006), Florence, Italy, September 4-8, 2006.

[14] Gu Tianming and Wang Yanjie, "DWT-based Digital image Watermarking Algorithm," 2011 10th International Conference on (Volume:3 ),pp.163-166, Aug 2011.

[15] Ikpyo Hong, Intaek Kim, and Seung-Soo Han “A Blind watermarking technique using Wavelet transform,” 2001. Proceedings. ISIE 2001. IEEE International Symposium on Industrial Electronics vol.3, pp.1946- 1950, June 2001.

[16] Huang Daren, Liu Jiufen, Huang Jiwu, Liu Hongmei, “A DWT based Image Watermarking Algorithm,” 2001 IEEE international conference on Multimedia and expo, , pp. 429-432,Aug 2001. 